\title{
Satisfação do paciente com degeneração macular relacionada à idade após terapia térmica transpupilar
}

\author{
Satisfaction of the patient with age-related maculardegeneration after transpupillary \\ thermotherapy
}

\author{
Jhony de Polo ${ }^{1}$ \\ Carlos Augusto Bastos ${ }^{1}$ \\ Alexandre Lass Siqueira² \\ Luciane Bugmann Moreira ${ }^{3}$ \\ Carlos Augusto Moreira ${ }^{4}$
}

\begin{tabular}{|l|}
\hline RESUMO \\
\hline Objetivo: Comparar resultados subjetivos (satisfação dos pacientes) aos \\
dados objetivos (melhora da acuidade visual - AV) após o tratamento de \\
degeneração macular relacionada à idade e/ou membrana neovascular sub- \\
retiniana por terapia térmica transpupilar. Métodos: Foram avaliados \\
retrospectivamente 23 prontuários de pacientes com diagnóstico angio- \\
fluresceinográfico de degeneração macular relacionada à idade submeti- \\
dos a uma única aplicação de terapia térmica transpupilar. Observou-se a \\
acuidade visual antes e um mês após a aplicação, e a satisfação dos \\
pacientes obtida com o tratamento. Resultados: Avaliamos 23 pacientes, \\
com idades variando entre 53 e 88 anos (média 74,08), dos quais 15 eram \\
do sexo feminino, e 8 do sexo masculino. Foram encontrados 12 olhos com \\
melhora da acuidade visual, 6 olhos sem alteração da acuidade visual, 6 \\
olhos com acuidade visual piorada. Em relação à satisfação, os resultados \\
obtidos foram: muito satisfeitos (4 olhos), satisfeitos (13 olhos), insatisfei- \\
tos (7 olhos) e ainda cruzando-se os dados obteve-se muito satisfeitos com \\
melhora da AV (3 olhos) e muito satisfeitos sem melhora da AV (1 olho); \\
satisfeitos com melhora da AV (7 olhos), satisfeitos sem melhora da AV (3 \\
olhos), e satisfeitos com piora da AV (3 olhos); insatisfeitos com melhora \\
da AV (2 olhos), insatisfeito sem melhora da AV (2 olhos), e insatisfeitos \\
com piora da AV (3 olhos). Conclusão: Em relação à acuidade visual, esta \\
foi melhorada em 12 olhos, dos 24 estudados; a quantidade de pacientes \\
muito satisfeitos ou satisfeitos atingiu a quantidade de 17 olhos, no \\
intervalo de um mês. Observando-se os resultados acima, pode-se consi- \\
derar a terapia térmica transpupilar como terapia alternativa ou adjuvante \\
para o tratamento de degeneração macular relacionada à idade.
\end{tabular}

Descritores: Degeneração macular/terapia; Neovascularização retiniana; Acuidade visual; Hipertermia induzida/métodos; Satisfação do paciente
Hospital Universitário Evangélico de Curitiba. Serviço de Oftalmologia

${ }^{1}$ Médico Residente do segundo ano de Oftalmologia do Serviço de Oftalmologia do Hospital Universitário Evangélico de Curitiba (HUEC)

2 Médico Oftalmologista.

3 Professora Adjunta da Faculdade Evangélica do Paraná. ${ }^{4}$ Professor Titular e Livre Docente da Faculdade Evangélica do Paraná e Universidade Federal do Paraná.

Endereço para correspondência: Jhony de Polo, Rua Desembargador Otávio do Amaral, 126 - Curitiba (PR) CEP 80730-400

E-mail: jhonydepolo@hotmail.com

Recebido para publicação em 06.03.2003

Versão revisada recebida em 17.09.2003

Aprovação em 12.11.2003

\begin{abstract}
INTRODUÇÃO
A terapia térmica transpupilar (TTT) é uma irradiação infravermelha de $810 \mathrm{~nm}$, produzida por meio de laser diiodado, causando hipertermia ${ }^{(1)}$, com baixa irradiação, foco largo e exposição prolongada ${ }^{(2)}$. Apesar de produzir calor, a temperatura atingida se mantém abaixo daquela requerida para fotocoagulação, causando menores danos à membrana de $\operatorname{Bruch}^{(1)}$. A TTT pode fechar neovascularização coroidea na degeneração macular relacionada à idade (DMRI) através de obliteração ou proliferação ${ }^{(2)}$. A TTT foi um método inicialmente criado para o tratamento de melanomas de coróide ${ }^{(1)}$, sendo hoje em dia usado para tratamento de membrana neovascular subretiniana (MNVSR), tanto como método único, quanto associado à terapia fotodinâmica (PDT) $)^{(1-2)}$.
\end{abstract}


A MNVSR é a principal causa de perda visual severa nos pacientes com DMRI e na miopia degenerativa ${ }^{(3)}$; podendo ser oculta ou clássica ${ }^{(2)}$; e é mais freqüentemente associada a DMRI, mas também pode ser secundária a outras causas, como a drusas de nervo óptico, doença de Gaucher, gravidez, uveítes e causas idiopáticas ${ }^{(4)}$. $\mathrm{O}$ crescimento de tecido fibrovascular proveniente da coróide, através da membrana de Bruch, para o espaço sub-retiniano (subepitélio pigmentar), constitui uma intercorrência fisiopatológica descrita em muitas doenças. Não se sabe até o momento quais são os mecanismos envolvidos na gênese destes quadros clínicos, contudo costuma-se implicar nos mesmos a participação de processos degenerativos, traumáticos, entre outros ${ }^{(3)}$. Na MNVSR ocorre alteração da anatomia macular - incluindo a interface fotorreceptora e o epitélio pigmentar da retina - permitindo o extravasamento de soro e/ou sangue e levando à perda irreversível dos fotorreceptores sobrejacentes ${ }^{(4)}$. Uma vez diagnosticados estes neovasos, quase sempre se associam à perda funcional significativa dentro de alguns meses, isto quando não são subfoveolares desde o início. Estes neovasos são o principal fator que leva os pacientes com DMRI a constituírem a principal causa de cegueira legal, acima de 50 anos, nos EUA e Europa ${ }^{(3)}$. Atualmente cerca de 25 a 30 milhões de pessoas são cegas devido à DMRI ${ }^{(5)}$. Considerando-se também o envelhecimento populacional decorrente do aumento da expectativa de vida, a DMRI pode tornar-se um importante problema de saúde pública nas próximas duas décadas, com sérias implicações socioeconômicas ${ }^{(5)}$.

O objetivo deste trabalho foi demonstrar resultados subjetivos (satisfação dos pacientes) e dados objetivos (acuidade visual - AV) após o tratamento de DMRI e/ou MNVSR por TTT.

\section{MÉTODOS}

Foi realizado um estudo retrospectivo de janeiro de 2001 até janeiro de 2002, no Hospital de Olhos do Paraná. Foram observados todos os pacientes submetidos a uma única aplicação de TTT, totalizando 23 pacientes (24 olhos), com diagnóstico angiofluresceinográfico de DMRI e/ou MNVSR. Resultados obtidos após uma subseqüente aplicação de TTT foram desconsiderados.

Para os dados objetivos observou-se acuidade visual (AV), medida na tabela de Snellen, antes e um mês após a aplicação de TTT. Graduou-se a AV em melhorada (quando o paciente apresentava um acréscimo na $\mathrm{AV}$ ), inalterada (AV sem alteração - persistência dos mesmos valores pré o pós TTT), e piorada (qualquer déficit de AV entre o pré e pós TTT). Comparou-se esses dados com a satisfação do paciente no mesmo período, dado subjetivo. A satisfação foi classificada em três categorias: muito satisfeito (MS), satisfeito (S) e insatisfeito (I) baseada na entrevista do paciente.

\begin{tabular}{|c|c|c|c|c|c|c|c|c|}
\hline & Sexo & Idade & AV pré TTT & AV pós TTT & Objetivo & Subjetivo & Olho op. & Doenca (DMRI)* \\
\hline 1 & $\mathrm{M}$ & 82 & $20 / 100$ & $20 / 100$ & Inalterada & MS & OD & MNVSR \\
\hline $2 a$ & $\mathrm{~F}$ & 74 & $\mathrm{Mm}$ & $\mathrm{Cd} 2,5 \mathrm{~m}$ & Melhorada & MS & OD & MNVSR \\
\hline $2 b$ & & & $\mathrm{Cd} 3,0 / \mathrm{m}$ & $\mathrm{Cd} 4,0 \mathrm{~m}$ & Melhorada & MS & OE & MNVSR \\
\hline 3 & $\mathrm{~F}$ & 88 & $\mathrm{Mm}$ & Cd 1,0m & Melhorada & I & $\mathrm{OE}$ & MNVSR \\
\hline 4 & $\mathrm{~F}$ & 80 & $\mathrm{Cd} 2,0 \mathrm{~m}$ & $20 / 200$ cc & Melhorada & $S$ & OE & DMRI \\
\hline 5 & $M$ & 81 & Cd $1,5 \mathrm{~m}$ & $20 / 200$ & Melhorada & MS & OD & MNVSR \\
\hline 6 & $M$ & 81 & $20 / 200$ cc & $20 / 60 \mathrm{cc}$ & Melhorada & $S$ & OD & MNVSR \\
\hline 7 & $\mathrm{~F}$ & 68 & $\mathrm{Cd} 3 \mathrm{~m}$ & $\mathrm{Cd} 2,0 \mathrm{~m}$ & Piorada & I & OD & DMRI \\
\hline 8 & $\mathrm{~F}$ & 72 & Cd $0,5 \mathrm{~m}$ & Cd $0,5 \mathrm{~m}$ & Inalterada & S & OE & MNVSR \\
\hline 9 & $\mathrm{~F}$ & 75 & $\mathrm{Cd} 0,5 \mathrm{~m}$ & $\mathrm{Cd} 2,0 \mathrm{~m}$ & Melhorada & $S$ & OD & DMRI \\
\hline 10 & $M$ & 63 & $\mathrm{Mm}$ & $\mathrm{Cd} 30 \mathrm{~cm}$ & Melhorada & I & $\mathrm{OE}$ & MNVSR \\
\hline 11 & M & 59 & $\mathrm{Cd} 2,0 \mathrm{~m} \mathrm{cc}$ & $\mathrm{Cd} 2,0 \mathrm{~m} \mathrm{cc}$ & Inalterada & I & OD & DMRI \\
\hline 12 & $\mathrm{~F}$ & 75 & $\mathrm{Cd} 1,0 \mathrm{~m}$ & $\mathrm{Mm}$ & Piorada & I & OD & DMRI \\
\hline 13 & M & 66 & $\mathrm{Cd} 0,5 \mathrm{~mm}$ & $\mathrm{Cd} 0,5 \mathrm{~m}$ & Inalterada & $S$ & OD & DMRI \\
\hline 14 & $\mathrm{~F}$ & 82 & $20 / 200$ & Cd 1,0m & Piorada & $S$ & OD & MNVSR \\
\hline 15 & $\mathrm{~F}$ & 78 & $\mathrm{Cd} 0,5 \mathrm{~m}$ & $\mathrm{Cd} 0,5 \mathrm{~m}$ & Inalterada & $S$ & OE & MNVSR \\
\hline 16 & M & 88 & $20 / 400$ cc & Cd 1,0m & Piorada & I & OD & DMRI \\
\hline 17 & $M$ & 58 & $\mathrm{Cd} 3,5 \mathrm{~m}$ & $\mathrm{Cd} 2,0 \mathrm{~m}$ & Piorada & $S$ & OE & MNVSR \\
\hline 18 & $\mathrm{~F}$ & 86 & $20 / 200 c c$ & $20 / 200 c c$ & Inalterada & $S$ & OE & MNVSR \\
\hline 19 & $\mathrm{~F}$ & 69 & $\mathrm{Cd} 1,0 \mathrm{~m} \mathrm{cc}$ & $20 / 100$ cc & Melhorada & $S$ & OD & DMRI \\
\hline 20 & $\mathrm{~F}$ & 53 & $\mathrm{Cd} 3,0 \mathrm{mcc}$ & $20 / 400$ & Melhorada & $S$ & OE & MNVSR \\
\hline 21 & $\mathrm{~F}$ & 67 & $\mathrm{Mm}$ & $\mathrm{Cd} 0,3 \mathrm{~m}$ & Melhorada & $S$ & OD & MNVSR \\
\hline 22 & $\mathrm{~F}$ & 82 & $\mathrm{Cd} 0,3 \mathrm{~m}$ & $\mathrm{Cd} 0,2 \mathrm{~m}$ & Piorada & $S$ & OE & MNVSR \\
\hline 23 & $\mathrm{~F}$ & 77 & Cd 1,5m & Cd $1,5 \mathrm{~m}$ & Inalterada & I & OE & MNVSR \\
\hline \multicolumn{9}{|c|}{$\begin{array}{l}\text { M = Masculino; } F=\text { Feminino; } A V=\text { Acuidade visual } ; T T T=\text { Terapia térmica transpupilar; } M m=\text { Movimentos de mão; } C d=\text { Conta dedos; } c c=\text { Com correção; } m=\text { Metros; } \\
\text { MS = Muito satisfeito; } S=\text { Satisfeito; I = Insatisfeito; OD = Olho direito; Olho esquerdo } \\
{ }^{*} \mathrm{DMRI}=\mathrm{DMRI} ; \mathrm{MNVSR}=\mathrm{DMRI}+\mathrm{MNVSR}\end{array}$} \\
\hline
\end{tabular}




\section{RESULTADOS}

Avaliou-se 23 pacientes (Tabela 1), com idades variando entre 53 e 88 anos (média 74,08), dos quais 15 eram do sexo feminino, e 8 do sexo masculino, totalizando-se 24 olhos. Foram encontrados 11 (45,8\%) olhos com melhora da acuidade visual, 7 (29,2\%) olhos sem alteração da acuidade visual, 6 (25\%) olhos com acuidade visual piorada, mostrando que a melhora da acuidade visual foi estatisticamente significativa $(\mathrm{p}=0,001)$ (Tabela 2). Em relação à entrevista, dado subjetivo, os resultados obtidos foram os seguintes: muito satisfeitos (4 olhos - 16,66\%), satisfeitos (13 olhos - 54,16\%), insatisfeitos (7 olhos - 29,16\%), mostrando que houve um bom nível de satisfação após o tratamento ( $\mathrm{p}=0,01$ ) (Tabela 3 ). Cruzando-se os dados obteve-se muito satisfeitos com melhora da AV (3 olhos - 12,50\%) e muito satisfeitos sem melhora da AV (1 olho - 4,16\%); satisfeitos com melhora da AV (6 olhos - 25,00\%), satisfeitos sem melhora da AV (4 olhos - 16,66\%), e satisfeitos com piora da AV (3 olhos 12,50\%); insatisfeitos com melhora da AV (2 olhos - 8,33\%), insatisfeito sem melhora da AV(2 olhos - 8,33\%), e insatisfeitos com piora da AV (3 olhos - 12,50\%). Após a análise estatística destes dados não se observou relação estatisticamente significativa entre a melhora da acuidade visual e o grau de satisfação dos pacientes ( $p=0,2149)$ (Tabelas 4 e 5).

\section{DISCUSSÃO}

A TTT pode ser usada não apenas como uma terapia única, mas também associada ao PDT, para o manejo da DMRI (degeneração macular relacionada à idade) $)^{(2)}$.

Em 1999 Reichel et al. publicaram o primeiro estudo sugerindo um novo tratamento para a forma oculta de MNVSR

\begin{tabular}{|lcc|}
\hline \multicolumn{3}{|c|}{ Tabela 2. Avaliação após o tratamento } \\
Avaliação & Número & Percentual \\
Igual & 7 & 29,2 \\
Melhor & 11 & 45,8 \\
Pior & 6 & 25,0 \\
Total & 24 & 100,0 \\
$\mathrm{z}_{\text {calc }}=3,175 ; p=0,001$ (Proporções) & \\
\hline
\end{tabular}

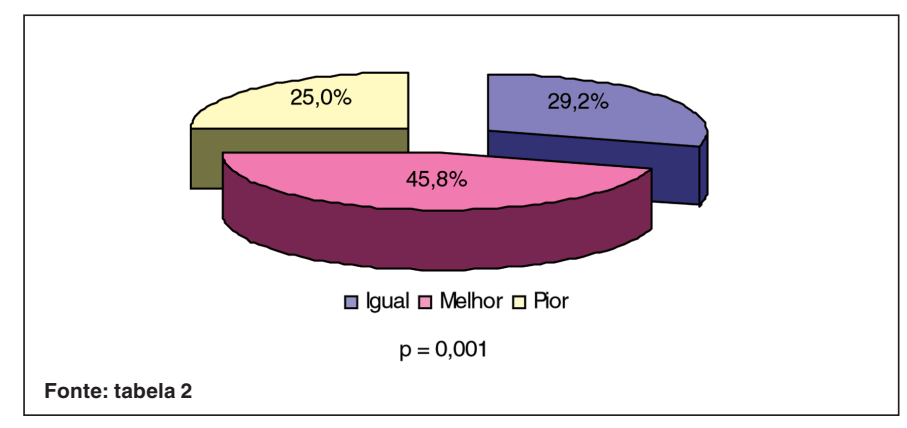

Gráfico 1 - Avaliação após o tratamento

\begin{tabular}{|lcc|}
\hline \multicolumn{2}{|c|}{ Tabela 3. Grau de satisfação dos pacientes após o tratamento } \\
Grau & Número & Percentual \\
Insatisfeitos & 7 & 29,2 \\
Satisfeitos & 13 & 54,2 \\
Muito Satisfeitos & 4 & 16,6 \\
Total & 24 & 100,0 \\
$z_{\text {calc }}=2,593 ; p=0,010$ (Proporções) & \\
\hline
\end{tabular}

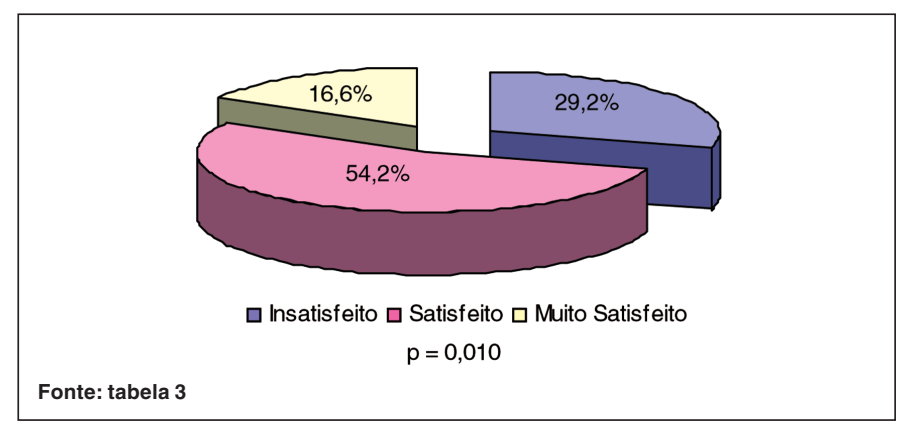

Gráfico 2 - Grau de satisfação dos pacientes após o tratamento

\begin{tabular}{|c|c|c|c|c|}
\hline \multirow{2}{*}{ Avaliação } & \multicolumn{2}{|c|}{ Insatisfeito } & \multicolumn{2}{|c|}{ Satisfeito } \\
\hline & $\mathrm{N}^{\circ}$ & $\%$ & $\mathrm{~N}^{\circ}$ & $\%$ \\
\hline Igual & 2 & 28,6 & 4 & 30,8 \\
\hline Melhor & 2 & 28,6 & 6 & 46,1 \\
\hline Pior & 3 & 42,8 & 3 & 23,1 \\
\hline Total & 7 & 100,0 & 13 & 100,0 \\
\hline \multirow{2}{*}{ Avaliação } & \multicolumn{2}{|c|}{ Muito } & \multicolumn{2}{|c|}{ Total } \\
\hline & $\mathbf{N}^{\circ}$ & $\%$ & $\mathbf{N}^{\circ}$ & $\%$ \\
\hline Igual & 1 & 25,0 & 7 & 29,2 \\
\hline Melhor & 3 & 75,0 & 11 & 45,8 \\
\hline Pior & - & - & 6 & 25,0 \\
\hline Total & 4 & 100,0 & 24 & 100,0 \\
\hline
\end{tabular}

secundária a DMRI, a $\mathrm{TTT}^{(4)}$. Uma das dificuldades desse tratamento é de se estabelecer a menor energia necessária para que o tratamento seja efetivo ${ }^{(4)}$.

Quanto a possíveis efeitos colaterais, apesar de existirem casos relatados de danos ao nervo ciliar, e ulceração de córnea após fotocoagulação de toda a retina, no uso da TTT para MNVSR as complicações são raras, e a tolerância é boa ${ }^{(6)}$. A membrana neovascular sub-retiniana clássica pode se tornar oculta após TTT devido, tanto a uma evolução natural, como devido a uma excessiva terapia ${ }^{(2)}$. Em nosso estudo não tivemos nenhuma complicação até o momento.

Em estudo prévio, analisando também o efeito da TTT em MNVSR oculta, associada à DMRI observou-se melhora da AV em 12\%, estabilização em 63\%, e piora em 25\% um mês após o tratamento ${ }^{(4)}$. Em um outro estudo, analisando o efeito da TTT em MNVSR clássica associada à DMRI, observou-se melhora da AV em 11\%, manutenção em 33\% e piora em 55\% dos olhos tratados ${ }^{(4)}$. 
Em outro estudo realizado, mostrou-se que a TTT foi efetiva para o fechamento de MNVSR, atingindo um valor de $70 \%$ das membranas fechadas após 6 meses, e apenas 7,1\% das membranas recorreram ${ }^{(6)}$, mostrando que a TTT pode ser considerada um tratamento potencial para MNVSR, mantendo a função visual, tanto nos pacientes com membrana clássica, quanto naqueles com membrana oculta ${ }^{(6)}$; além de apresentar custo relativamente baixo e ausência de riscos sistêmicos ${ }^{(7)}$.

Nossos resultados (45,8\% de melhora de AV, e 70,82\% de pacientes muito satisfeitos, ou satisfeitos); estão em sintonia com diversos estudos. Um não randomizado mostrou visão estabilizada ou melhorada em $75 \%{ }^{(6)}$. Dentre os pacientes citados na tabela 2, os 3 pacientes com piora da AV que relatavam subjetivamente melhora referiam que o escotoma tinha um aspecto melhor, ou seja, de escuro tinha se tornado claro. Nos pacientes com melhora da AV que referiam piora do quadro, acreditamos que isto tenha ocorrido devido à expectativa de uma melhora significativa da visão a qual não ocorreu. Outro estudo constatou, ao longo de 50 meses que, 20\% dos seus doentes com DMRI mantinham o quadro inalterado ${ }^{(3)}$. Encontramos $25 \%$ de nossos pacientes sem alterações da AV após 1 mês de TTT.

\section{CONCLUSÃO}

Em nosso estudo a acuidade visual foi melhorada em 11 dos 24 olhos estudados; a quantidade de pacientes muito satisfeitos ou satisfeitos atingiu 17 olhos, dados estes que nos levam a considerar a TTT como uma possível alternativa ao tratamento de DMRI, apesar de salientarmos que estes resultados obtidos são no intervalo de um mês de seguimento. Estudos com maior número de casos e seguimento mais prolongado são necessários para nos fornecer mais informações sobre esse tratamento.

\section{ABSTRACT}

Purpose: Comparison of subjective outcome (patient's satisfaction) with objective data (improvement of visual acuity-VA) after treatment of age-related macular degeneration (ARMD). Methods: In a retrospective study we analyzed 23 charts of patients with angiofluoresceinographic diagnosis of agerelated macular degeneration submitted to only one applica- tion of transpupillary thermotherapy (TTT). Visual acuity was recorded before and one month after the application, and the satisfaction of the patients with the treatment. Results: 23 patients (15 females and 8 males), 53 to 88 years old (mean 74.8) were evaluated. 12 eyes were found with improvement of visual acuity, 6 eyes without alteration of visual acuity, 6 eyes with worse visual acuity. Regarding satisfaction the results were: 4 eyes, very satisfied; 13 eyes, satisfied; 7 eyes, not satisfed, and checking the data we found 3 eyes, very satisfied with the improvement of visual acuity and 1 eye, very satisfied without improvement of VA. Satisfied with improvement of visual acuity there were 7 eyes, satisfied without improvement of VA, 3 eyes and satisfied with worsening of VA, 3 eyes. Conclusion: Visual acuity was improved in 12 eyes of the 24 studied eyes; the number of very satisfied patients reached 17 eyes within one month. Observing these results transpupillary thermotherapy may be considered as alternative or adjuvant treatment for age-related macular degeneration.

Keywords: Macular degeneration/therapy; Retinal neovascularization; Visual acuity; Hyperthermia, induced/methods; Patient satisfaction

\section{REFERÊNCIAS}

1. Currie ZI, Rennie IG, Talbot JF. Retinal vascular changes associated with transpupillary thermotherapy for choroidal melanomas. Retina 2000;20:620-6.

2. Mainster MA, Reichel E. Transpupillary thermotherapy for age-related macular degeneration: long pulse photocoagulation, apoptosis, and heat shock proteins. Ophthalmic Surg Lasers 2000;31:359-73.

3. Vilela M, Corrêa-Meyer G, Corrêa-Meyer R. Neovascularização sub-retiniana: opções atuais de manejo. Rev Méd Sta Casa P Alegre 1994;5:1091-4.

4. Nehemy M, Rodrigues RP, Campos C, Passos E, Magalhães E. Tratamento de membrana neovascular sub-retiniana idiopática ou secundária à miopia patológica, a estrias angióides e à distrofia padrão tipo asa de borboleta pela temoterapia transpupilar. Rev Bras Oftalmol 2001;60:319-27.

5. Verma L, Das T, Binder S, Heriot WJ, Kirshhof B, Venkatesh T et al. New approaches in the manegement of choroidal neovascular membrane in agerelated macular degeneration. Indian J Ophthalmol 2000;48:263-78.

6. Newsom RS, MacAlister JC, Saeed M, MacHugh JD. Transpupillary thermotherapy (TTT) for the treatment of choroidal neovascularization. Br J Ophthalmol 2001;85:173-8.

7. Nehemy M, Passos E, Campos C, Rodrigues RP, Nehemy DP. Indocianina verde como adjuvante da termoterapia transpupilar em membrana neovascular sub-retiniana secundária à degeneração macular relacionada à idade. Rev Bras Oftalmol 2001;60:251-9.

8. Rapizzi E, Grizzard WS, Caponi Jr A. Transpupillary thermotherapy in the manegement of circumscribed choroidal hemangioma. Am J Ophthalmol 1999;127:481-2.

\section{Ao enviar um artigo para publicaçáo, leia ATENTAMENTE as instruções parca autores, constante no final de cada fascículo.}

